\title{
Prospective Applications of Microwaves in Medicine
}

\author{
Jaroslav Vorlíček, Barbora Vrbova and Jan Vrba \\ Czech Technical University \\ Czech Republic
}

\section{Introduction}

Research of interactions between Electromagnetic (EM) Field and biological systems is of growing interests elsewhere. In this area of research very important activity are studies of prospective medical applications of microwaves (i.e. a possibility to use microwave energy and/or microwave technique and technology for treatment purposes) are a quite new and a very rapidly developing field. Microwave thermotherapy is being used in medicine for the cancer treatment and for some other diseases since early eighties. Most common methods used for treatment of cancer are radiotherapy and chemotherapy. These therapeutic methods have many undesirable effects, such as usage of ionizing radiation. Another method used for treatment of cancer is hyperthermia. This method is based on the principle of destruction of malignant cells by artificially increased temperature in the temperature range between 41 and $45{ }^{\circ} \mathrm{C}$ fails self-protective mechanism of malignant cells. In contrast with chemotherapy and radiotherapy, hyperthermia is not limited by the number of doses of radiation.

\section{Prospective applications of microwaves in medicine}

In the following text we would like to outline scope and new trends in medical applications of microwaves. We can divide these results and new trends into two major groups:

- clinical results and trends,

- technical results and trends.

\subsection{Clinical results and trends}

Applications of microwaves in medicine is a quite a new field of a high interest in the world (since early $80^{\prime} \mathrm{s}$ ). It is necessary to mention one of the most important trends in the research of medical applications of microwaves, i.e. the thermal effects of EM field (since early 80's a microwave thermotherapy is used for cancer treatment, Benign Prostate Hyperplasia (BPH) treatment and for some other areas of medicine; it can be used in combination with other complementary treatment methods also.

To give a basic overview, we can divide medical applications of microwaves in following three basic groups according to purpose, how are microwaves used:

- Microwave energy and/or technique used for the treatment of patients (with the use of either thermal or non-thermal effects - sometimes both of these types of effects can play its role). 
- Microwave energy and/or technique used for diagnostics of diseases (e.g. by aid of permittivity measurements, attenuation measurements and very prospective in the near future can be a microwave tomography).

- Microwave energy and/or technique used as a part of a treatment or diagnostic system (e.g.linear accelerator).

As is given above, until now medical applications of microwaves are above all represented by the treatment methods based on thermal effect - i.e. we can speak about the Microwave thermotherapy, which can be further divided into three different modalities distinguished according to the goal temperature level or interval:

- diathermia: heating up to $41 \mathrm{C}$

(applications in physiotherapy)

- $\quad$ hyperthermia: heating into the interval of 41-45 C (applications in oncology)

- thermodestruction / thermoablation: over 45 C (applications in urology)

First three of the following list of thermotherapeutical applications are just largely used in many countries around the world, last three instead are in this moment in the phase of very promising projects:

$\begin{array}{lll}\text { - } & \text { Oncology } & \text { - cancer treatment. } \\ \text { - } & \text { Physiotherapy } & \text { - treatment of rheumatic diseases. } \\ \text { - } & \text { Urology } & \text { - BPH treatment. } \\ \text { - } & \text { Cardiology } & \text { - heart stimulations. } \\ \text { - } & \text { Surgery } & \text { - growing implants. } \\ \text { - } & \text { Ophthalmology } & \text { - retina corrections. }\end{array}$

E.g. in cancer treatment is thermotherapy usually used in the combination with some of other modalities used in the clinical oncology (e.g. radiotherapy, chemotherapy, immunotherapy or chirurgical treatment). It is used in USA, Japan and in many countries in European Union (EU), including the Czech Republic, from early 80s. Up to now local microwave hyperthermia for cancer treatment and thermotherapy of $\mathrm{BPH}$ are the most important applications of microwaves in medicine. In the Czech Republic we have treated more than 500 patients. Results of our treatments are given the following table:

\begin{tabular}{|l|l|}
\hline Complete response of the tumor & $52.4 \%$ \\
\hline Partial response of the tumor & $31.7 \%$ \\
\hline Without response & $15.9 \%$ \\
\hline
\end{tabular}

Table 1. Clinical results of cancer treatment by radiothermotherapy

Successful treatment thus has been indicated in the case of $84 \%$ of patients. This corresponds very well to the results published in EU and USA. Actual scientific information about microwave thermotherapy and its new developments is possible to get from "International Journal of Hyperthermia“ issued by European Society for Hyperthermia Oncology (ESHO) together with North American Hyperthermia Society (NAHS) and Asian Society of Hyperthermia Oncology (ASHO). On the following series of photographs (Fig. 1) there is example of the typical 4 phases of the treatment of one of our patients obtained by radio-thermo-therapy (i.e. combination of microwave hyperthermia with significantly reduced doses of radiotherapy).

Very interesting results for the near future can give us the research of microwave medical applications based on so called non-thermal effects. In the literature it is possible just now to identify research projects on: 
- $\quad$ to reduce pain feeling (analogically to analgesics),

- to increase of reaction capabilities of a human being,

- to examine the teaching capabilities, etc.

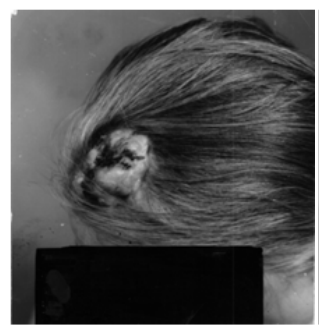

a)

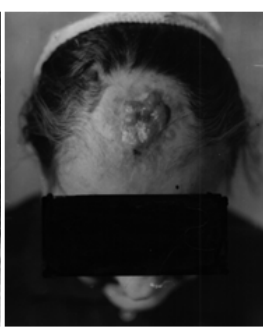

b)

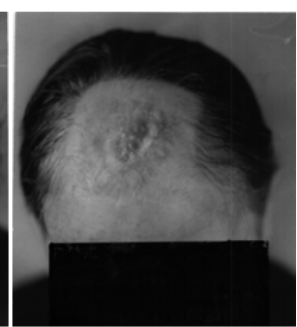

c)

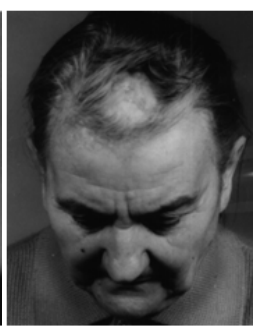

d)

Fig. 1. Typical example of 4 phases of the patient treatment: a) Patient before the treatment. b) At the end of the treatment. c) Two months after treatment. d) One year after treatment.

\subsection{Technical results and trends}

Our work is focused on the design, optimization and tests of the microwave applicators for medical applications, above all for hyperthermia cancer and $\mathrm{BPH}$ treatment. This means to design a microwave structure capable to:

- transfer EM energy into the biological tissue without power reflections back to the microwave generator,

- guarantee the best possible approximation of the area to be treated by the 3D distribution (coverage) of Specific Absorption Rate (SAR).

Our applicators (working at $434 \mathrm{MHz}$ and at $2450 \mathrm{MHz}$ ) were used for the treatment of more than 500 patients with superficial or subcutaneous tumour. Now, following new trends in this field, we continue our research in the important directions of deep local and regional applicators. Most important technical fields of microwave thermotherapy development (covered also by our activities) can be specified as:

- Applicators: development and optimization of new applicators for more effective local, intracavitary and regional treatment,

- Treatment planning: mathematical and experimental modelling of the effective treatment

- Non-invasive temperature measurement: research of the possibilities of new techniques like Magnetic Resonance Imaging (MRI) and Ultrasound (US) for exact non-invasive measurements.

- $\quad$ Microwave medical diagnostics - e.g. Microwave Tomography (MT).

\section{Applicators for microwave thermotherapy}

Simulations of SAR distribution obtained by array of microwave applicators radiating at frequency $434 \mathrm{MHz}$ will be described here. We will compare here the SAR distribution in a homogeneous agar phantom (which has similar dielectric characteristics to muscle tissue) and in an anatomical based biological model, which has been developed from a Computer Tomography (CT) scan. 


\subsection{Waveguide applicators}

Wave guide applicators are based on microwave waveguide technology. Major advantage of waveguide applicator is that EM field in theirs aperture is roughly at $50 \%$ of its area very near to the case of EM plane wave, which is the wave with the best homogeneity and gives us the deepest penetration into the treated area.

Mostly rectangular and circular waveguides are being used to build microwave thermotherapy applicator. Examples of mentioned applicators are given in the Fig. 2. Typically they are operating at $434 \mathrm{MHz}$ (depth of efficient treatment is between 4 and $6 \mathrm{~cm}$ ), in case of deeper treatment lower frequency must be selected. Waveguide applicators displayed in Fig. 2a. are filled by dielectric material in order to decrease their cut off frequency. Air filled Evanescent mode applicator (Fig. 2b.) is excited under its cut-off frequency.

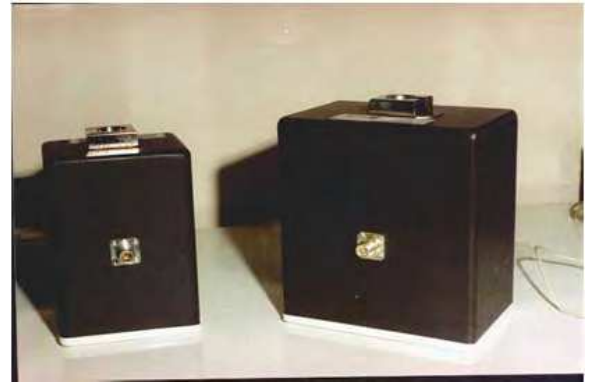

a)

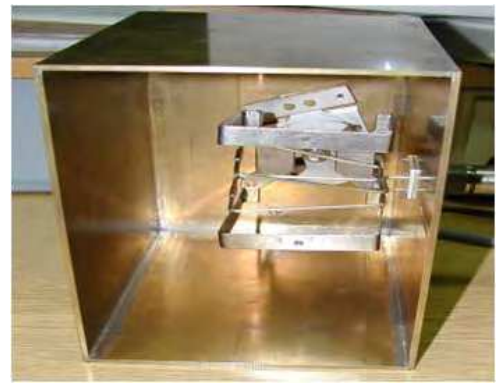

b)

Fig. 2. a) Dielectric filled waveguide applicator, b) air filled Evanescent mode applicator.

On the next picture (Fig. 3.) there is a sketch of evanescent waveguide mode applicator working at $70 \mathrm{MHz}$, including its SAR distribution. There is a water bolus between aperture of the applicator and the agar phantom mimicking the biological tissue.

Interesting possibility how to achieve relatively sharp beam from external applicator is to use a focusing principle. The aperture of a standard rectangular waveguide applicator is then divided into 3 or 5 sectors with shifted excitation (i.e. different amplitude and phase). Another way how to focus microwave power is to use an array of external applicators, where amplitudes and phases of excitation of single applicators can be used for electronic steering of the focus area.

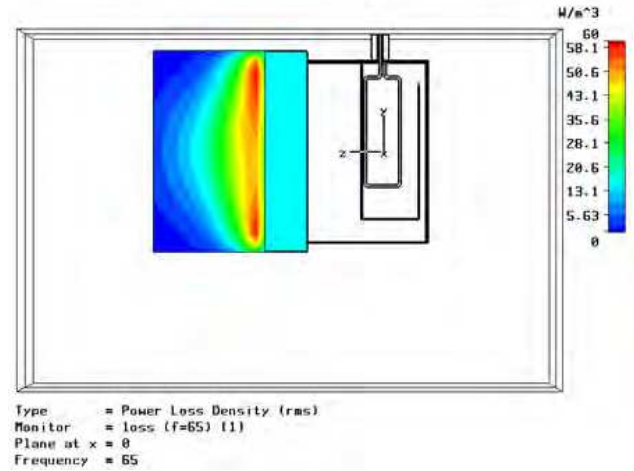

Fig. 3. Evanescent mode applicator and its calculated SAR pattern 


\subsection{Microwave stripline TEM applicators}

Microwave stripline applicator with TEM (Transversal Electromagnetic Mode) is being described in the following. The TEM wave propagation depends on dielectric parameters, such as permittivity $\varepsilon$ and permeability $\mu$. The upper and bottom part of applicator consists of high conductivity material (copper). Transversal dimensions of the described applicator are $50 \times 30 \mathrm{~mm}$. Distance of exciting probe from short circuited section is $15 \mathrm{~mm}$. Lateral sides of applicator are made from dielectric material, from acrylic glass, which is $2 \mathrm{~mm}$ thick. The second part of applicator consists of stripline horn. Dimensions of the horn aperture are $120 \times 80 \mathrm{~mm}$ and length of applicator equals to two wavelengths. Wavelength depends on permittivity of environment (in our case $\varepsilon_{\mathrm{H} 20}=78$ ). Whereas $70 \%$ of human body consists of water, applicator is filled with water. Advantage of this is better transfer of EM energy into human body and smaller dimensions of the applicator. This applicator was designed by the Finite Difference Time Domain (FDTD) simulator EM Field simulator (e.g. SEMCAD X).

\subsection{Deep local heating applicators}

Waveguide type applicators are very suitable for the deep local thermotherapy treatment based either on the principle of dielectric filled waveguide or on the principle of evanescent modes (i.e. waveguides excited below its cut-off frequency) - which is our specific solution and original contribution to the theory of microwave hyperthermia applicators. Technology of evanescent mode applicators enable us to design applicators with as small aperture as necessary also for relatively low frequencies e.g. from 10 to $100 \mathrm{MHz}$, needed for deep penetration into the biological tissue (i.e. up to 10 centimetres under the body surface). In theoretical and experimental evaluation, the grade of homogeneity of the temperature distribution in the target area has been tested, see the Fig. 4. Our mathematical approach is based on idea of waveguide $\mathrm{TM}_{01}$ (Transverse Magnetic) mode excited in the agar phantom under the given conditions (see the dashed lines). Measurement of SAR (full lines) has been done on agar phantom of the muscle tissue.

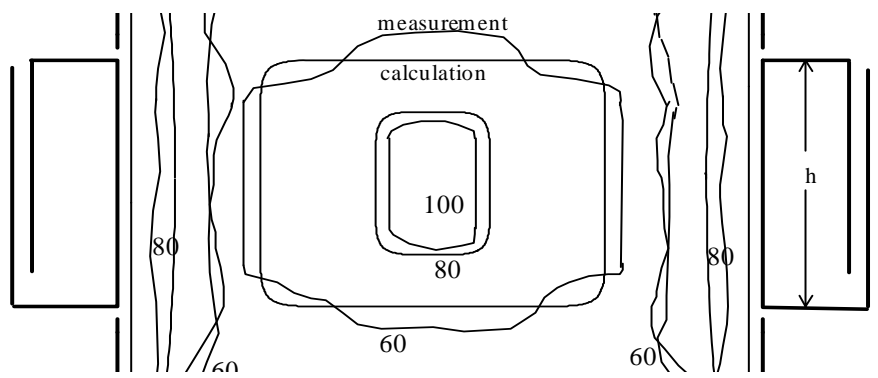

Fig. 4. Normalized SAR distribution (both calculated (full line) and measured (dashed line)) in the agar phantom.

\subsection{New principles for the regional applicators}

Goal of this work is the development of the new applicators with higher treatment effects. Methodological approach for the solution of the problem will be theoretical and experimental study of the new types of regional applicators. Our aim is to improve the present theoretical model to optimize the temperature distribution in the treated area. 
We can design the regional applicators on the basis of the analytical description of the excited electromagnetic field for the case of simplified homogeneous model of the treated biological tissue. For a more realistic case of the non-homogeneous dielectric composition of the biological tissue the analytical calculation does not guarantee enough exact results. Therefore we would need to build the equipment for experimental tests of the applicators and we will study the possibilities of its numerical mathematical modelling.

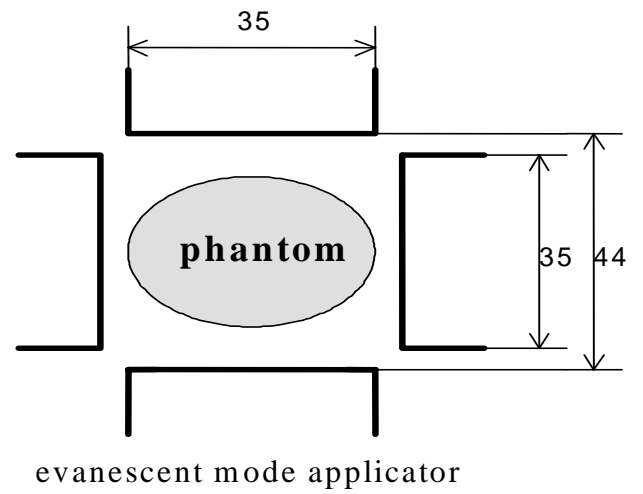

Fig. 5. Set up of proposed regional applicator (4 evanescent mode waveguides applicators)

Applicators must have perfect impedance matching for good protection of microwave generator against reflected power. For measurement of impedance matching a reflection parameter $\mathrm{s}_{11}$ is mostly used. This parameter shows, how much electromagnetic power is reflected back to generator. Electromagnetic field is inside discussed applicator excited at frequency of $434 \mathrm{MHz}$.

\subsection{Anatomical model}

To verify basic functionality of created applicator we can use a homogeneous agar phantom. This homogeneous phantom represents only one type of tissue in human body - in our case it represents muscle tissue. For further discussion we will use cylindrical agar phantom with diameter $8 \mathrm{~cm}$, which can basically represent a human limb. In reality human body is very inhomogeneous, and therefore it is for hyperthermia treatment planning necessary to use anatomical precise 3D models with all types of biological tissues taken into account. 3D model thus can be created from segmentation of series of several scans from CT or MRI. Such scans are 2 dimensional transversal gray - scale cuts. After then is created 3D model imported to the program, which is used for hyperthermia treatment planning. This program must both cooperate well with a segmentation program and in the same moment to enable calculation of SAR distribution.

We can choose e.g. Woman's calf (Fig. 6.) as an example to demonstrate EM simulations potential. Given anatomical model was created by segmentation program 3D - DOCTOR (vector-based 3D imaging, modelling and measurement software (Able Software Corp., 2011)). We should have available DICOM scans from CT (MRRF, 2010). DICOM scans hold the original quality of data. Model of woman's calf has resolution of $1 \mathrm{~mm}$ and mean voxel size is $1 \times 1 \times 1 \mathrm{~mm}$. 


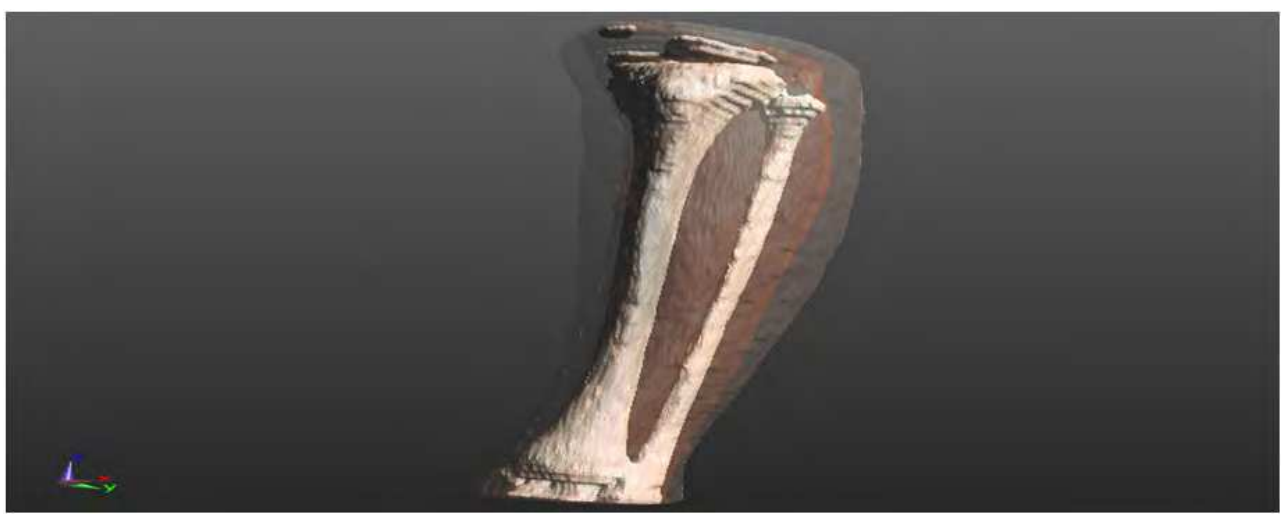

Fig. 6. 3D anatomical model of woman's calf

Each voxel was assigned to one of 3 different types of biological tissue, such as bone, muscle and fat with dielectric parameter shown in table (Table 1.).

\begin{tabular}{|l|l|l|}
\hline Name & Conductivity [S/m] & Relative Permittivity [-] \\
\hline Agar & 0.80 & 54.00 \\
\hline Bone Cortical & 0.09 & 13.07 \\
\hline Muscle & 0.80 & 56.86 \\
\hline Fat & 0.04 & 5.56 \\
\hline
\end{tabular}

Table 2. Dielectric properties at frequency $434 \mathrm{MHz}$

\subsection{Array of applicators}

EM behaviour of arrays of 2, 3 and 4 stripline type applicators coupled to homogeneous cylindrical agar phantom and consequently on 3D anatomical model of woman's calf. Cylindrical agar phantom represents muscle tissue of human limb. In our case we simulated muscle tissue of woman's calf with diameter of $8 \mathrm{~cm}$. The 3D anatomical model consists of 3 various types of tissue of woman's calf created from CT scans. All of here discussed arrays of applicators were simulated by FTDT program SEMCAD X.

\subsubsection{Array of $\mathbf{2}$ microwave stripline applicators}

Case of array of 2 applicators on cylindrical agar phantom coupled to 3D anatomical model of woman's calf is shown on following picture (Fig. 7.). From both normalized SAR distributions (Fig. 8.) it follows that this array of 2 applicators is suitable for treatment of tumor, which cover larger area near to surface of human limb.

\subsubsection{Array of $\mathbf{3}$ microwave stripline applicators}

The other possible configuration is array of 3 applicators coupled to homogeneous cylindrical agar phantom (Fig. 9a.). Consequently, we studied simulations of 3 applicators coupled to the 3D anatomical model (Fig. 9b.).

Another interesting case is shown on Fig. 10. -in this picture there are displayed interesting shapes of SAR distribution by change of phases of applicators and change of amplitude of one type of applicator located in the middle of all applicators. 


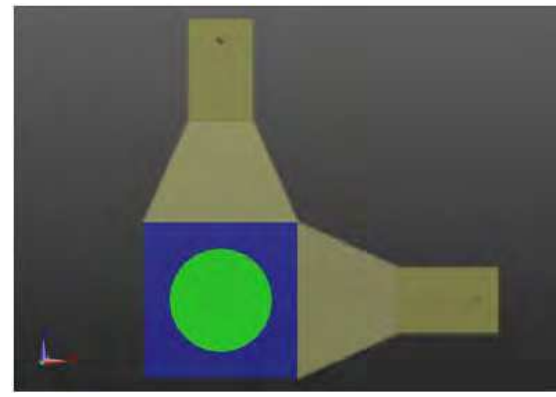

a)

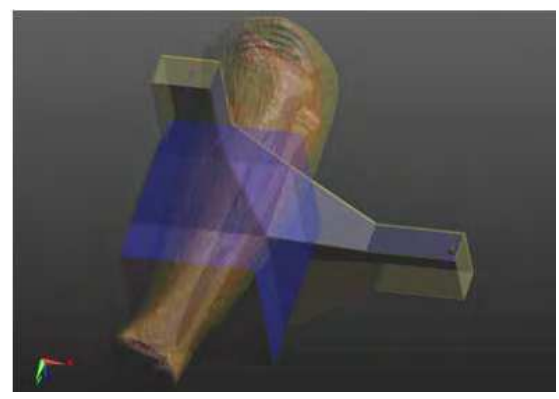

b)

Fig. 7. Array of 2 applicators a) on cylindrical agar phantom, b) on anatomical model

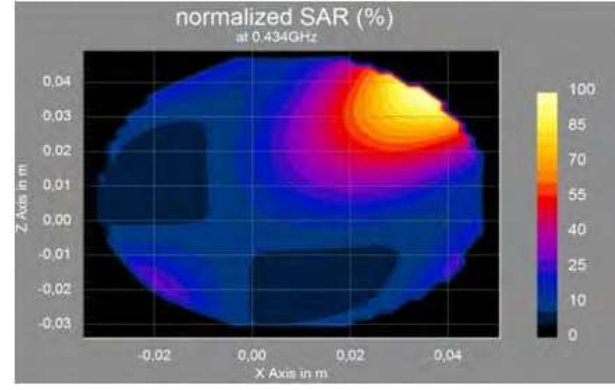

a)

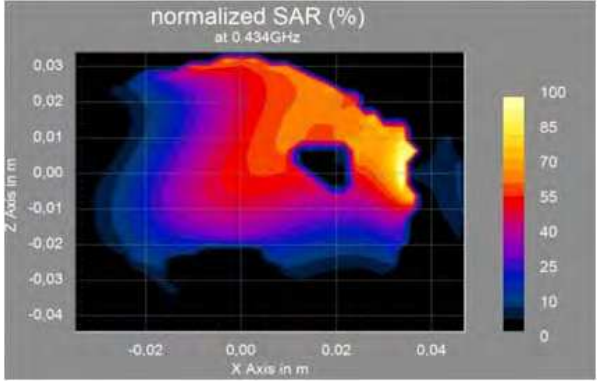

b)

Fig. 8. Normalized SAR a) in agar phantom, b) in anatomical model

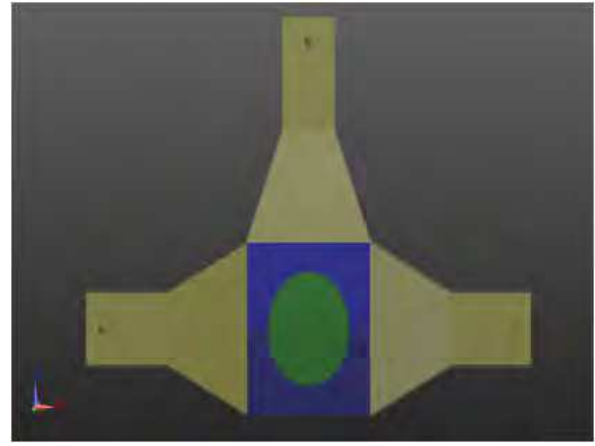

a)

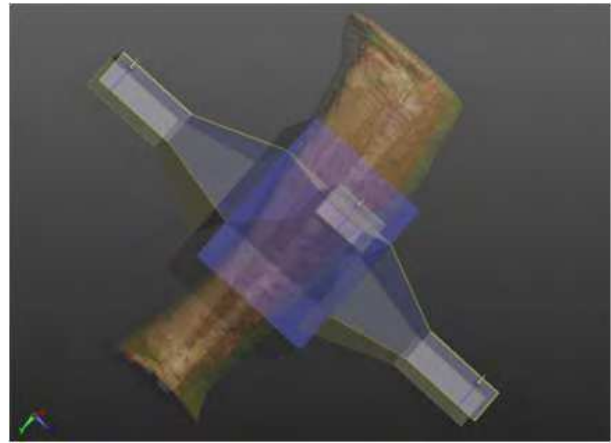

b)

Fig. 9. Composition of 3 applicators a) on cylindrical agar phantom, b) on anatomical model

In homogeneous phantom were created 3 maxima, instead in anatomical model we can observe only one maximum of SAR distribution - that demonstrates very nice example of focussing possibilities. By setting of phases and/or amplitude of applicators we can accomplish required shape of SAR. 


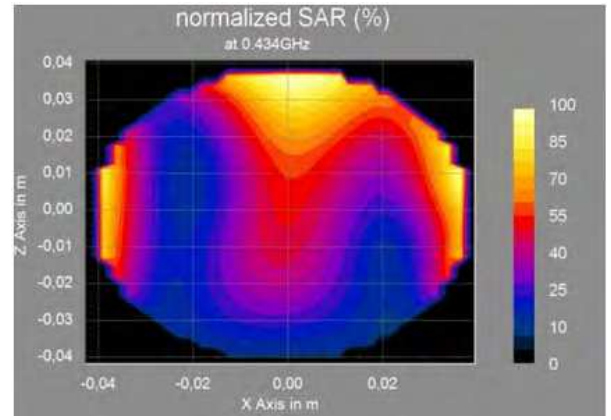

a)

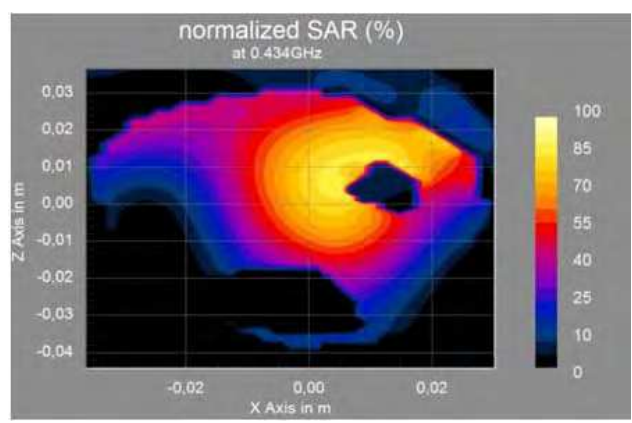

b)

Fig. 10. Normalized SAR a) in agar phantom, b) in normalized SAR in anatomical model

\subsubsection{Array of $\mathbf{4}$ microwave stripline applicators}

By using 4 applicators we can see in following picture (Fig. 11.), that in both cases there is a very good focusing effect of SAR distribution near to the cylindrical axis.

By alternation of phases of several applicators we achieved better focusing in the middle of both homogeneous agar phantom and 3D anatomical model (Fig. 12.). Such array of 4 applicators could be used for treatment tumors located in the middle of human limb.

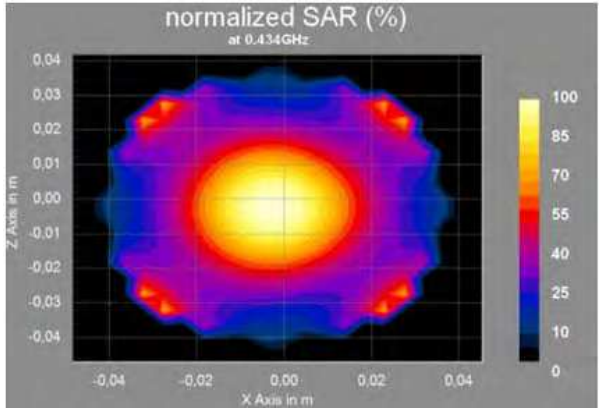

a)

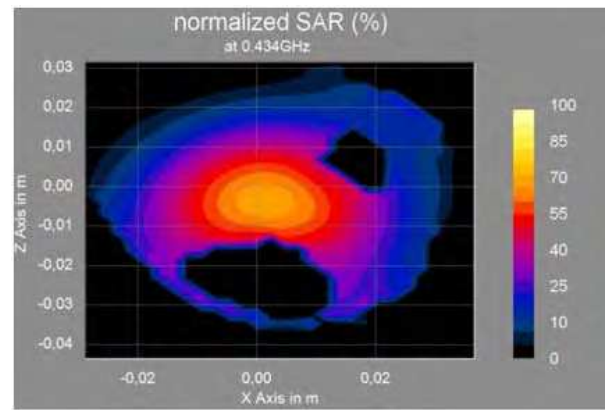

b)

Fig. 12. Normalized SAR a) in agar phantom, b) in anatomical model

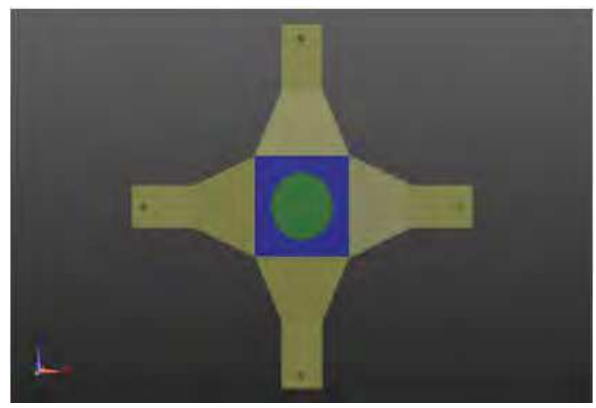

a)

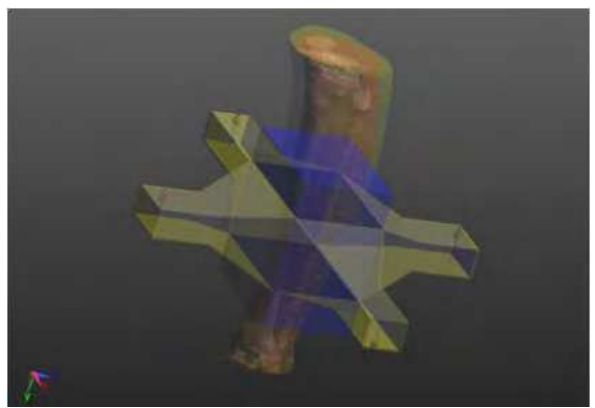

b)

Fig. 11. Array of 4 applicators a) on cylindrical agar phantom, b) on anatomical model 


\section{Microwave applicators for „BPH“ thermotherapy}

In all above mentioned key activities we need to do numerical simulations of SAR and temperature 3D distribution. Temperature distribution inside area of biological tissue heated by microwave energy can be calculated from well known formula:

$$
\rho_{t} c_{t} \frac{\partial T}{\partial t}=\gamma_{t} \Delta T-\chi\left(\mathrm{T}-\mathrm{T}_{\mathrm{b}}\right)+\mathrm{q}
$$

where $t$ is the time.

$q=q(x, y, z, t)$

$T=T(x, y, z, t)$

$T b=T b\left(x, y, z, t_{0}\right)$

is energy delivered by EM field, is designates the temperature, is the temperature of blood,

Physical meaning and values of the here used constants for the case of high water contents tissue are:

$\mathrm{r}_{\mathrm{t}}=0.965[\mathrm{~g} / \mathrm{cm} 3] \quad$ is density of biological tissue $(\mathrm{BT})$.

$\mathrm{c}_{\mathrm{t}}=3586[\mathrm{~mJ} / \mathrm{g} / \mathrm{C}] \quad$ is specific heat of BT.

$\mathrm{k}=5.45[\mathrm{~mW} / \mathrm{cm} 3 / \mathrm{C}] \quad$ is blood flow and temperature capacity of BT.

$\mathrm{g}_{\mathrm{t}}=5.84[\mathrm{~mW} / \mathrm{cm} / \mathrm{C}] \quad$ is spec. temp. conductivity of BT.

Possibilities of analytical solution of this equation are very limited to only a few cases - like e.g. "one dimensional" case of plane wave penetrating in homogeneous phantom. Therefore computers are to be used to solve this equation to obtain the temperature $T(x, y, z, t)$ time dependence and space distribution. For the treatment planning of microwave thermotherapy we use software product SEMCAD. Fig. 13 gives basic idea about SAR calculated for the case of the monopole intracavitary applicator.

\subsection{Applicators for „BPH“ treatment}

We have investigated basic types of microwave intracavitary applicators suitable for $\mathrm{BPH}$ treatment, i.e. monopole, dipole and a helical coil structures. These applicators are designed to work either at 915 or at $434 \mathrm{MHz}$. We would like to discuss its effective heating depth, based on the comparison of the theoretical and experimental results. Basic mechanisms and parameters influencing (limiting) heating effective depth are described and explained in ref. (Vrba et al., 1996, 1999).

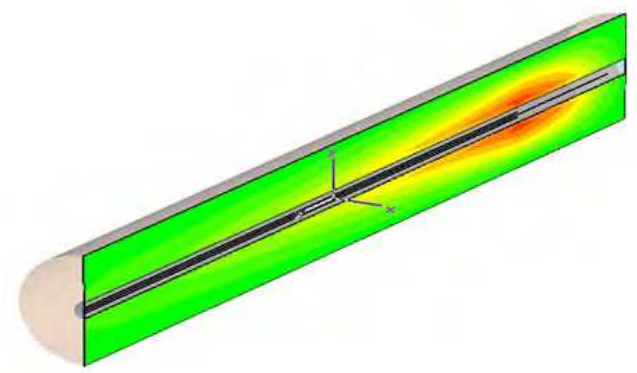

Fig. 13. Calculated SAR of BPH applicator

The basic type of intracavitary applicator is a monopole applicator. The construction of this applicator is very simple, but calculated and measured SAR distribution along the 
applicator is more complicated, than has been the first idea. SAR can be measured either in water phantom or by infrared camera.

\subsection{Evaluation of BPH applicators}

One of our tools for experimental evaluation of microwave applicators is the apparatus, which enable us to do 3D measurements of SAR distribution. It can be used for both local external and intracavitary applicators.

The basic part of this apparatus is big salt water phantom (water with $0.3 \%$ to $0.6 \% \mathrm{NaCl}$ ) and the measurement probe with the possibility of 3D scan around the applicators. As probes we use a Light Emitting Diodes (LED) with a fibber optic link connection to interface of the computer. The purpose of this link is to reduce influence of the metallic (i.e. conductive) components from the measured electromagnetic field. The schematics of the apparatus for 3D measurements of SAR distribution is shown in Fig. 14.

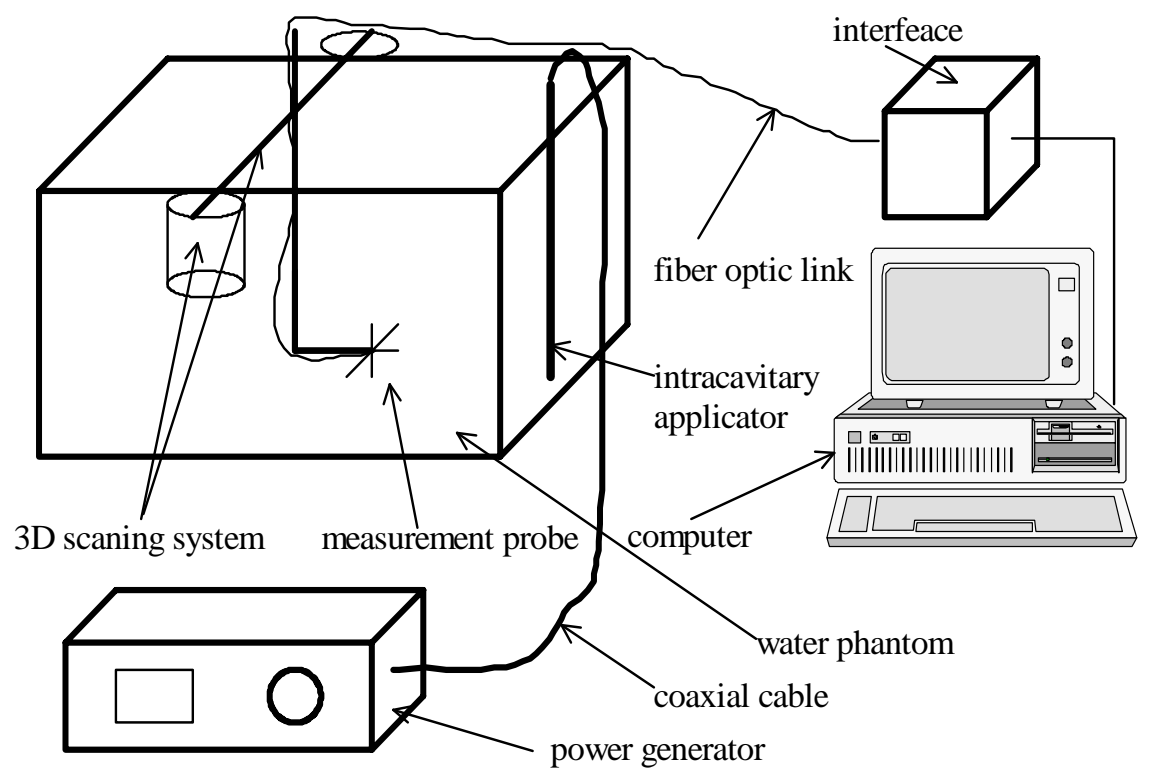

Fig. 14. Apparatus for 3D SAR measurements

During measurements of SAR along the applicator we have found, that typically there is not only a one main SAR maximum (first from the right side), but also a second and/or higher order maxima can be created, being produced by outside back wave propagating along the coaxial cable, see Fig. 15a. In Fig. 15b. SAR distribution improvement (i.e. reduction of second maximum) can be noticed for the case of dipole like applicator. To eliminate this second maximum and optimise the focusing of SAR in predetermined area of biological tissue needs to use the helical coil antenna structure.

After coil radius and length optimisation we have obtained very good results of SAR distribution, see Fig. 16a,b. Some problems can be with the antenna self-heating, but it can be reduced by cooler at the end of applicator tip. 


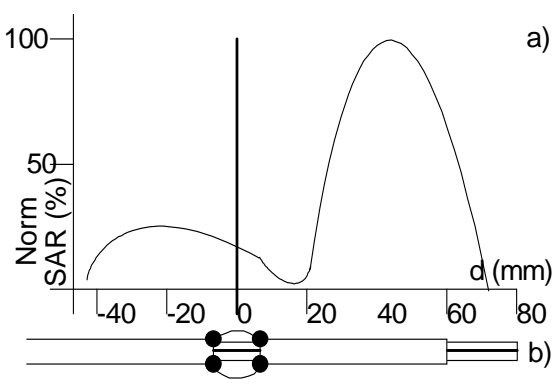

a)

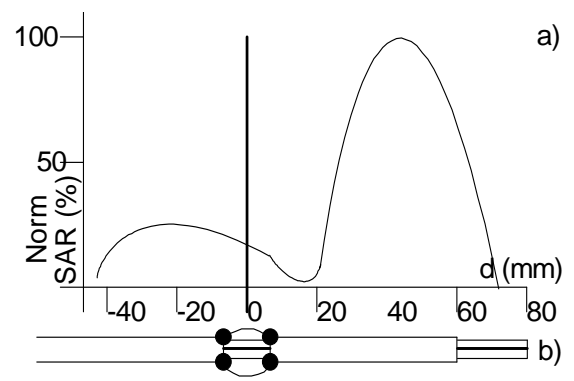

b)

Fig. 15. a) Monopole applicator, b) Dipole applicator

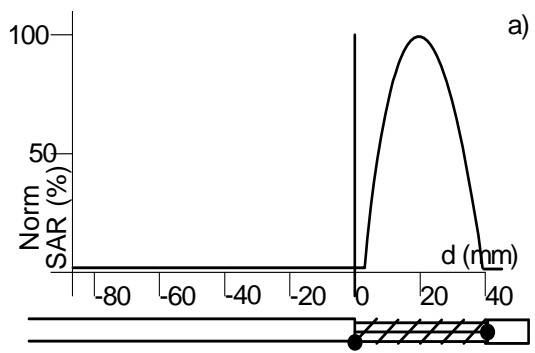

b)

a)

)

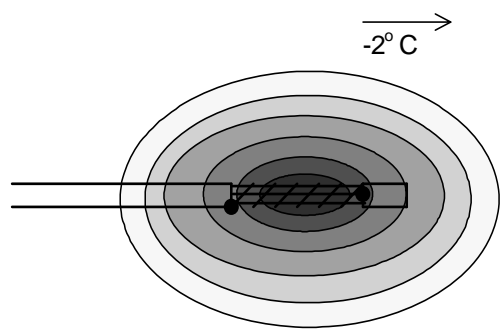

$20 \mathrm{~mm}$

b)

Fig. 16. a) Helical coil applicator, b) Temperature field around the helix

\subsection{Effective treatment depth}

We have studied the theoretical limits of intracavitary applicator heating depth. We have found the basic relation for determination of the limit of maximum heating depth for the case of "very long" intracavitary applicator. We suppose excitation of an ideal radial wave around radiating applicator.

Mentioned results can be simply interpreted by following figure, where on the horizontal axis is the working frequency of thermotherapy apparatus and on vertical axis there is effective depth of electromagnetic wave penetration. As a third parameter playing an important role there is a diameter of a discussed intracavitary applicator.

Very important is that in this case we are dealing with a radial wave, not the plane wave, and that's why the penetration depth is smaller than penetration depth of plane wave. Some works published in this field give too optimistic results. Measurements discussed without theoretical analysis can give results influenced by thermal conductivity of mostly used agar phantom of muscle tissue. As the real heating depth is typically a few millimetres (in the best case up to approx. $1 \mathrm{~cm}$ under the surface of the cavity), thermal conductivity of the phantom material can easily cause errors of several tenth of percents.

Ideal intracavitary applicator irradiates an ideal cylindrical wave into the biological tissue surrounding the cavity. According to our experience Fig. 17. gives very good 
approximation, i.e. the results with accuracy better than $5 \%$ for higher frequencies (f $>100$ $\mathrm{MHz}$ ) and/or bigger radii $(\mathrm{R}>3 \mathrm{~mm}$ ). For lower frequencies (up to $100 \mathrm{MHz}$ ) combined with small radius of the cavity $(\mathrm{R}<2 \mathrm{~mm}$ ) the accuracy is cca $10 \%$. It is not possible to achieve a heating penetration depth (i.e. $50 \%$ decrease) higher than $R$ at any frequency and in any propagation medium. The small cavity radius plays a dominant role in the penetration depth.

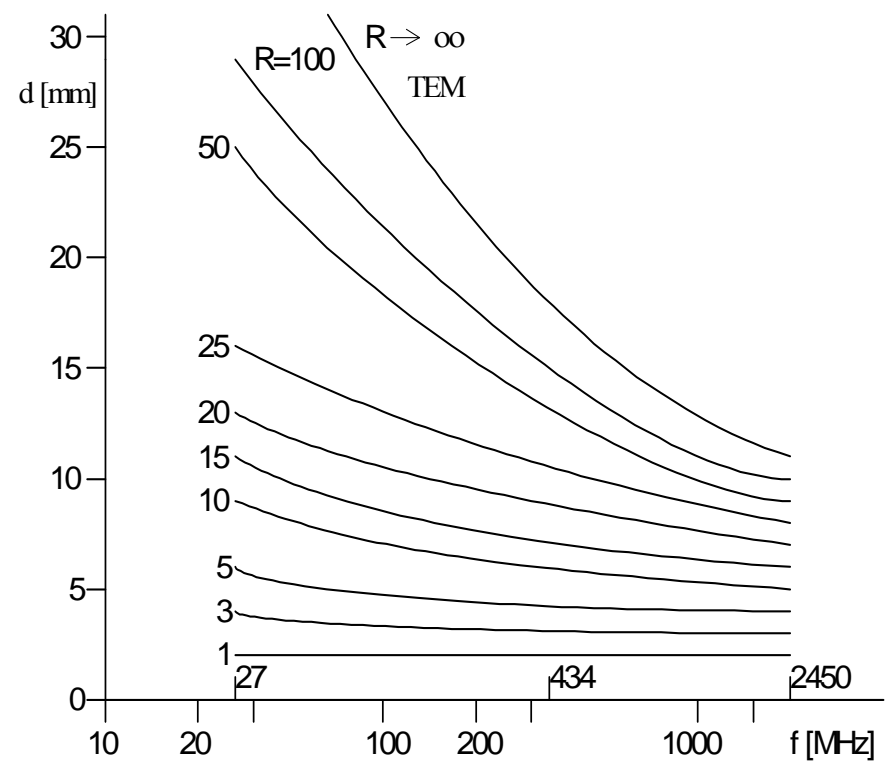

Fig. 17. Effective depth of heating $d$ with respect to frequency $f[\mathrm{MHz}]$ and radius $\mathrm{R}[\mathrm{mm}]$

\subsection{SAR measurements by infrared camera}

Infrared camera is according to our experiences a very efficient tool for "SAR" measurements of microwave intracavitary applicators. In Fig. 18. there is the typical measured heating pattern of monopole (a), dipole (b), and helical coil antennas (c). This pattern can be obtained by heating suitable phantoms of biological tissue - mimicring the dielectric and thermal properties of the prostate tissue. Such phantoms can be made on the basis of agar or so called superstuff material. The pattern is colour-coded according to a linearly decreasing scale of white-yellow-red, where white is the maximum temperature. A diagrammatic catheter is inserted in each figure; the orientation of the bladder neck in a patient is indicated by a dashed line. Note the long back heating tails with a monopole antenna (Fig. 18a.) which is caused by microwave currents that flow backwards along the cable and cause the feeding cable to radiate. The radiation pattern from a dipole antenna (Fig. 18b.) is generally well confined without any excessive back heating. The dipole antenna is suitable for prostates with axial length $>40 \mathrm{~mm}$. The helical coil antenna (Fig. 18c.) has the shortest and most focussed heating and would be the choice for small prostates, $25-40 \mathrm{~mm}$ in length. 


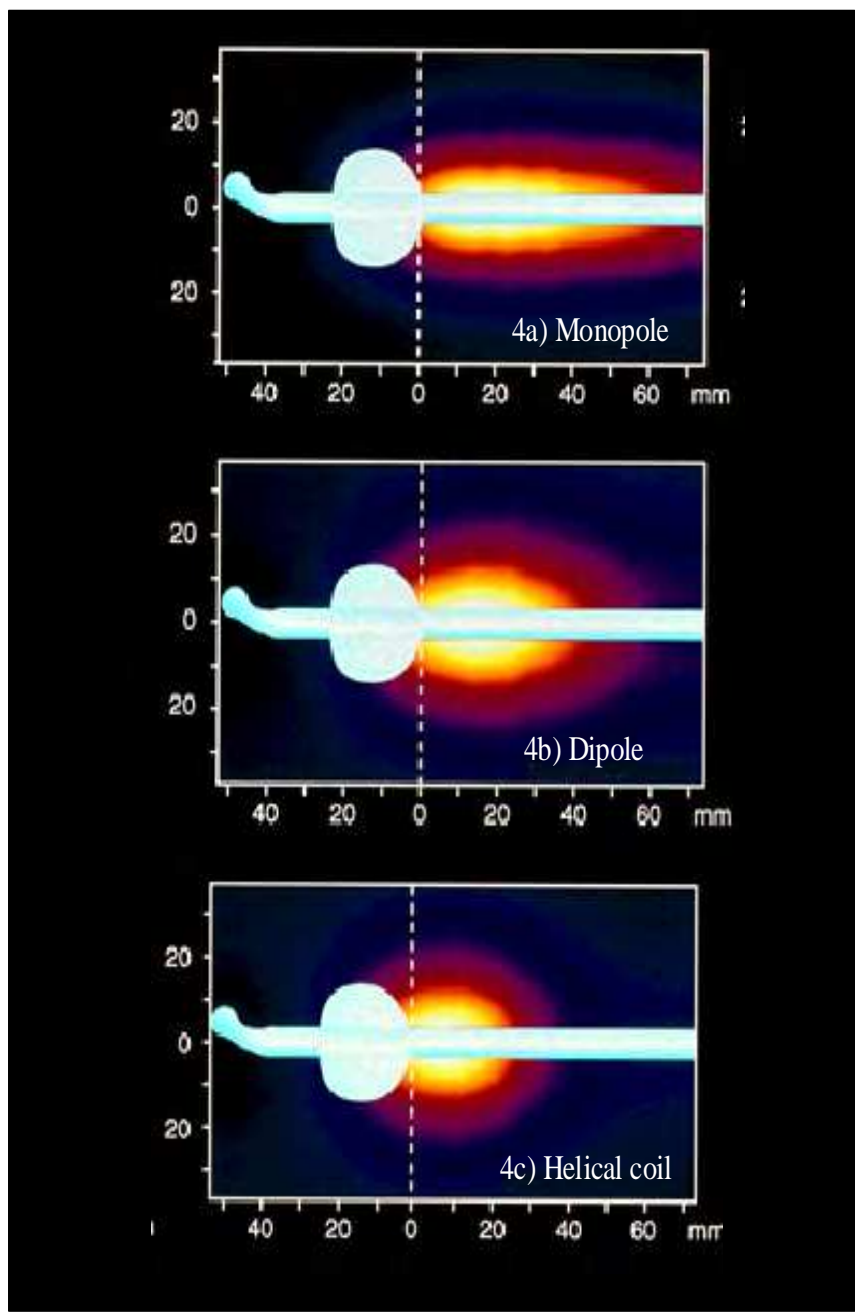

Fig. 18. The heating pattern for different antennas: a) the monopole, b) the dipole, c) the helical coil.

The pattern is colour-coded according to a linearly decreasing scale of white-yellow-red, where white is the maximum temperature. A diagrammatic catheter is inserted in each figure; the orientation of the bladder neck in a patient is indicated by a dashed line.

The pattern is colour-coded according to a linearly decreasing scale of white-yellow-red, where white is the maximum temperature. A diagrammatic catheter is inserted in each figure; the orientation of the bladder neck in a patient is indicated by a dashed line.

Moreover, by using infra-red camera we can follow a lot of other properties resp. behaviour of intracavitary applicators itself, like e.g. standing waves along feeding coaxial cable (i.e. the risk of existence of unwanted SAR and/or temperature maxima), possible overheating of some specific parts of tested applicator itself etc. 


\section{Technical equipment for research of biological effects of EM field}

In present time four research institutions here in the Czech Republic run research projects focused on studies of interactions between EM field and biological systems. These institutions are technically supported by Dept. of EM Field of the Czech Technical University in Prague. In this contribution we would like to give more details about that projects and obtained technical results (i.e. description of developed exposition systems).

Three of discussed projects ( 1 in Germany and 2 here in Czech Republic) are basic research for simulation of the microwave hyperthermia treatment. Other two projects (both in Czech Republic are focused on simulation of the case of exposition by mobile phone.

In the modern view, cancer is intended as a complex illness, involving the cells that undergo to transformation, their environment, and the general responses at biochemical and biological levels induced in the host. Consequently, the anti-cancer treatment protocols need to be multi-modal to reach curative effects. Especially after the technical improvements achieved in the last 15 years by bio-medical engineering, microscopy devices, and molecular biology methods, the combinations of therapeutic procedures are growing in interest in basic and clinical research.

The combination of applied biological research together to the physical sciences can offer important perspectives in anticancer therapy (e.g. different methodologies and technical devices for application of energies to pathological tissues).

The modern bioengineering knowledge applied to traditional tools, as the microscopy, has largely renewed and expanded the fields of their applications (e.g.: in vivo imaging), pushing the interest for direct morpho-functional investigations of the biomedical problems.

\subsection{Waveguide applicator}

Very good results of EM field expositions in biological experiments can be obtained by simple but efficient waveguide applicators see example in Fig. 19. Waveguide offer a very big advantage - in approximately of fifty percents of its aperture the irradiated electromagnetic field is very near to a plane wave, which is basic assumption for good homogeneity of the heating and optimal treatment penetration.

Here described system is being used (shared) for research projects by two institutions (Institute of Radiation Oncology in Prague and Institute of Microbiology of the Czech Academy of Sciences).

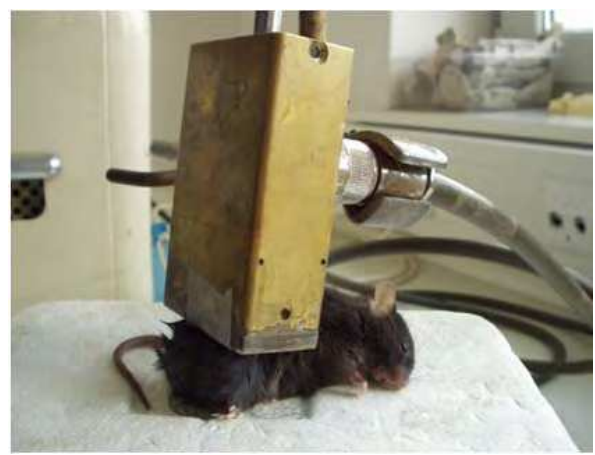

Fig. 19. Waveguide applicator for biological experiments 
Aperture of this waveguide is $4.8 \times 2.4 \mathrm{~cm}$ and it is excited at frequency $2.45 \mathrm{GHz}$. Effective heating is in the middle of the real aperture - its size is approximately $2.4 \times 2.4 \mathrm{~cm}$. Waveguide is filled by teflon to reduce its cut-off frequency. Power from generator is possible to control from 10 to $180 \mathrm{~W}$, in these experiments we work between 10 and $20 \mathrm{~W}$ mostly.

\subsection{Evaluation of waveguide applicator}

To evaluate this applicator from technical point of view we made a series of experiments, see e.g. Fig. 20, where you can see example of measurement of temperature distribution by IR camera.

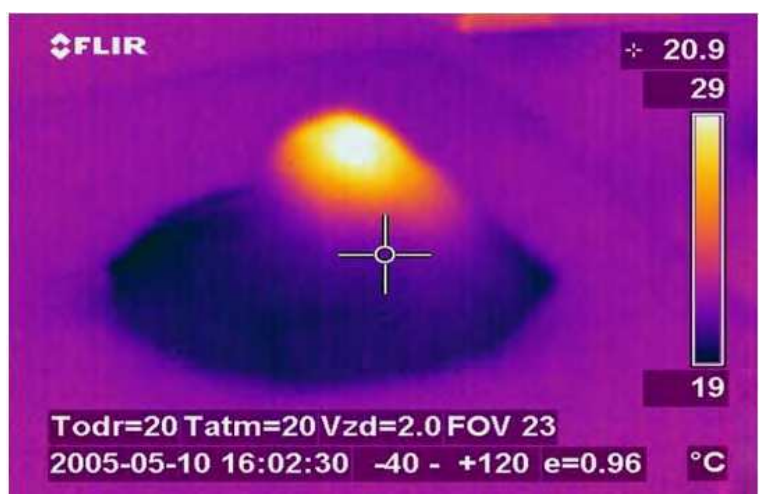

Fig. 20. Temperature distribution obtained on surface of a model of mouse

Here you can see temperature distribution obtained on surface of a model of mouse made from agar - with a simulated tumour on mouse back. Experiment has been done by heating phantom during 2 minutes delivering a power of $10 \mathrm{~W}$. Maximum of temperature increase has been found approximately $10{ }^{\circ} \mathrm{C}$. Similar results with different increase in temperature we have got also in other technical experiments on phantom or live mouse when power or heating time was changed.

Next Fig. 21. gives example of temperature increase on the surface of the phantom and $1 \mathrm{~cm}$ in that phantom. Temperature was measured by our 4-chanel thermometer. In this case with two thermo probes. Heating here is scheduled to 9 times repeated $30 \mathrm{~s}$ of heating and $30 \mathrm{~s}$ pause. Difference in temperature on the surface and under it is on the level of $1{ }^{\circ} \mathrm{C}$. That means very good homogeneity of temperature distribution in the treated area during planned biological experiments.

\subsection{Array applicator}

The main goal of the planned biological experiment is a hyperthermia treatment of the experimentally induced pedicle tumours of the rat to verify the feasibility of ultrasound diagnostics and magnetic resonance imaging respectively to map the temperature distribution in the target area of the treatment. That means to heat effective volume of approximately cylindrical shape (diameter approx. $2 \mathrm{~cm}$, height approx. $3 \mathrm{~cm}$ ). Temperature to be reached is $41^{\circ} \mathrm{C}$ or more (i.e. temperature increase of at least $4{ }^{\circ} \mathrm{C}$ from starting point $37^{\circ} \mathrm{C}$ ), time period of heating is 45 minutes. 


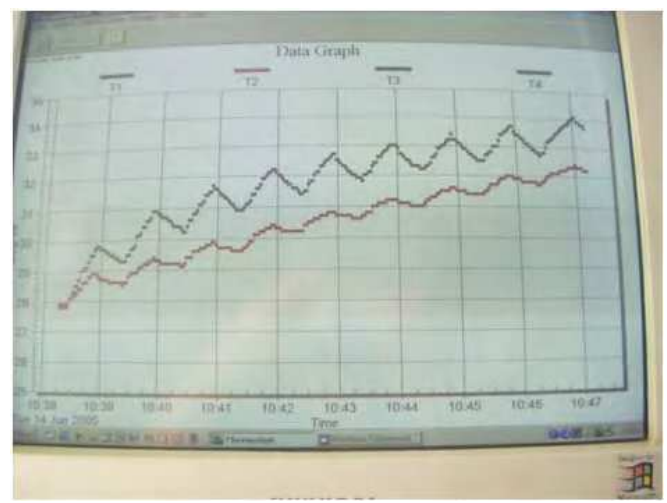

Fig. 21. Temperatures during experiments

Considering the necessary effective heating depth for the planned experiments, we have found $915 \mathrm{MHz}$ to be suitable frequency. As an excellent compatibility of the applicator with non-invasive temperature measurement system (ultrasound or NMR) is a fundamental condition for our project, we should have to use non-magnetic metallic sheets of minimised dimensions to create the conductive elements of the applicator. Therefore the applicator itself (see Fig. 22.) is created by two inductive loops tuned to resonance by capacitive elements (Vrba, 1993). Dimensions of these resonant loops were designed by our software, developed for this purpose. Coupling between coaxial feeder and resonant loops (not shown in Fig. 22.) as well as a mutual coupling between resonating loops could be adjusted to optimum by microwave network analyser.

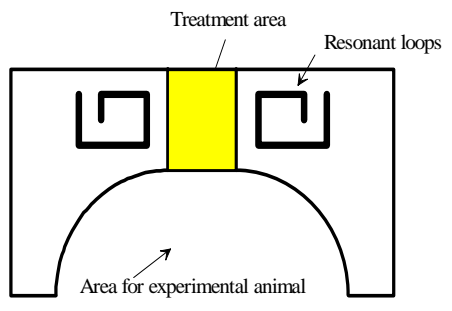

a)

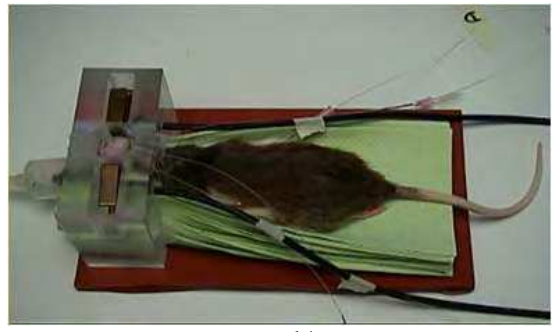

b)

Fig. 22. a) Arrangement of discussed microwave hyperthermia applicator, b) Photograph of the discussed applicator

The position of the loops is fixed by perspex holder. There is a special cylindrical space for experimental animal in lower part of this perspex holder. As the heated tissue has a high dielectric losses, both loops are very well separated and so no significant resonance in heated area can occur. From this follows, that either the position of the loops with respect to heated area or the distance between the loops is not very critical.

First measurements to evaluate the basic properties of the discussed applicator were done on agar phantom of muscle tissue:

- evaluation of basic microwave properties (transfer of EM energy to the tissue, reflections), 
- evaluation of compatibility with US and NMR,

- calculation and measurement of SAR and temperature distribution and its homogeneity.

Exact tuning of the resonant loops to frequency $915 \mathrm{MHz}$ has been easy and we could optimise the coupling between the coaxial feeders and resonant loops as well, reflection coefficient less than 0.1 . We have tested the power to be delivered to the applicator to obtain sufficient temperature increase (approximately $4{ }^{\circ} \mathrm{C}$ in less than 5 minutes is required). With power $10 \mathrm{~W}$ delivered to each loop for period of 2 minutes we succeeded to obtain the temperature increase of approximately $7^{\circ} \mathrm{C}$. To keep the increased temperature for a long time, $2 \mathrm{~W}$ in each loops were sufficient. Similar values were obtained during first experiments on rats also. Even with higher level of delivered microwave power we did not observe the change of resonant frequency (caused by increased temperature of the loops). This applicator has been developed for German Cancer Research Institute in Heidelberg. And it is being used there for a series of animal experiments to study effect of hyperthermia on tumours and possibility to combine hyperthermia with chemotherapy etc.

Compatibility of this applicator with a Magnetic resonance unit (MR) has been studied and it has been demonstrated. We have tested the influence of the applicators on US diagnostics and NMR imaging and the result of this evaluation shows very good compatibility. Only a negligible deterioration of the US images has been observed when the incident power was kept under $100 \mathrm{~W}$.

Details about influence of microwave power on MR imaging are given in Fig. 23. We can see here a sequence of images of the discussed applicator made by MR unit for four different cases. First case (upper left) is image for the case without power excitation of the applicator. Second case (left down) a power of $10 \mathrm{~W}$ has been delivered to each loop. We can see quite clear configuration of the applicator set-up. Third case (upper right) gives situation when 20 $\mathrm{W}$ has been delivered to each loop. Slight noise but still quite a clear configuration of the applicator set-up can be observed. Fourths case (right down) gives situation when $40 \mathrm{~W}$ has been delivered to each loop. In this case noise disturbed the possibility to observe the configuration of the applicator.

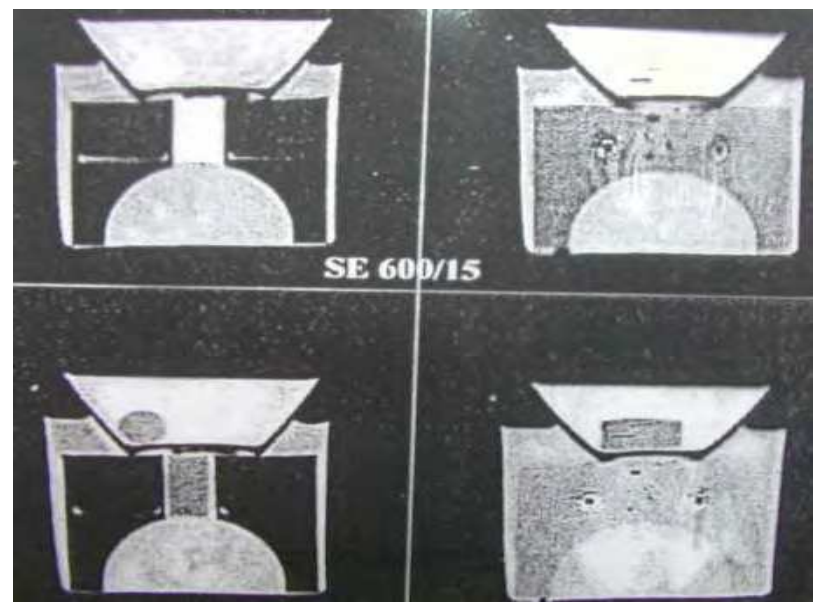

Fig. 23. MR images of the discussed applicator 
In Fig. 25. we can see temperature vs. time measurement in the case of agar phantom inserted in the studied applicator. Next Fig. 26. shows experimental setup of applicator and simple exposure chambers installed at Medical Faculty of Charles University in Pilsen. 3D distribution of SAR distribution was verified numerically (Fig. 24).

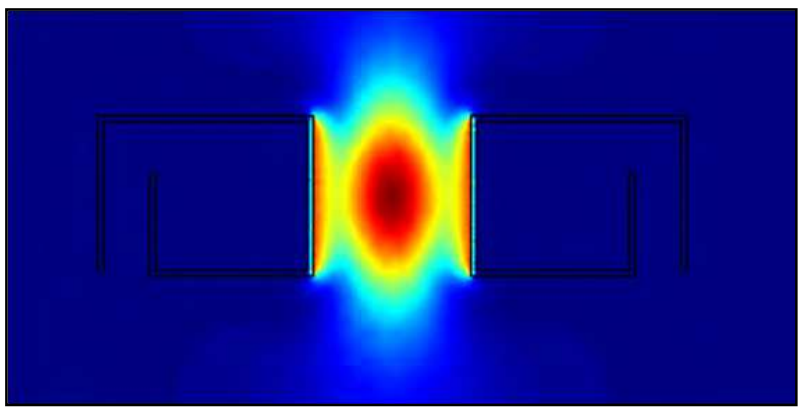

Fig. 24. Numerical SAR analysis.

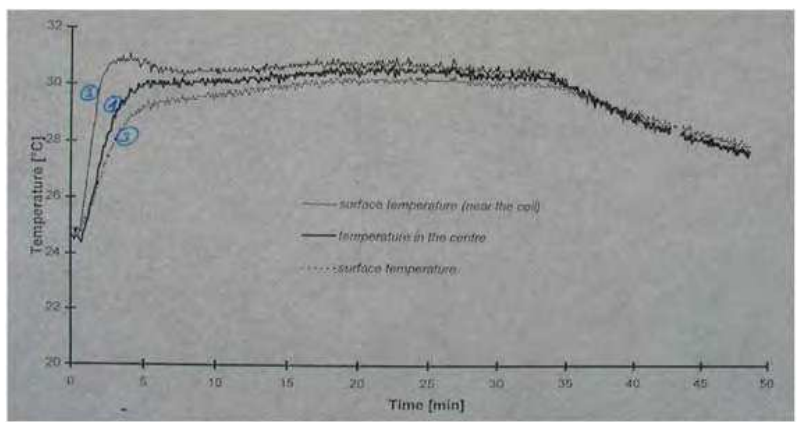

Fig. 25. Temperature measurements

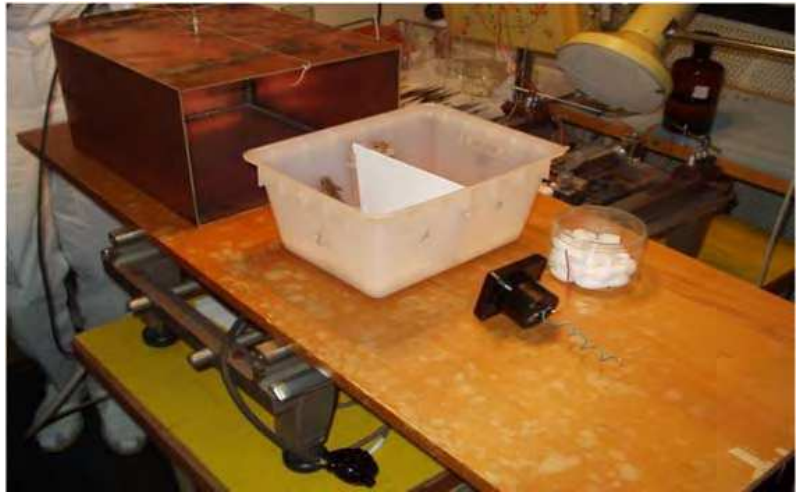

Fig. 26. Exposure system for research of electromagnetic field and biological system interactions 


\section{Measurement of the complex permittivity of the biological tissue as the possible imaging method}

\subsection{Introduction}

This sub-chapter describes and evaluates a method for determining complex permittivity, and presents results of permittivity measurement of inhomogeneous agar phantom and living tissue. There are described different approaches for measurement of complex permittivity, in particular the non-invasive method of measuring complex permittivity at the end of the coaxial cable. Vector measurement of the reflection coefficient on the interface between probes and measured samples is performed with the aid of network analyzer in the frequency range from $300 \mathrm{kHz}$ to $2 \mathrm{GHz}$. The results indicate that using the coaxial probe with dimensions of SMA (subminiature version A) connector is suitable in the frequency range approximately from $300 \mathrm{MHz}$ to $10 \mathrm{GHz}$. In order to demonstrate the diagnostic potential of this method, measurements were first conducted on artificially created inhomogeneous agar phantom with added mixture of various dielectrics, followed by measurement of living biological tissue.

\subsection{Complex permittivity}

Relative permittivity, loss factor and conductivity are basic parameters for electromagnetic field modeling and simulations. Although these parameters could be found in the tables for many materials, their experimental determination is very often necessary (Hippel, 1954). Dielectric properties of biological tissues are determining factors for the dissipation of electromagnetic energy in the human body and therefore they are useful in hyperthermia cancer treatment. Measurement of the dielectric parameters of biological tissues is also a promising method in the medical diagnostics and imaging. Knowledge of the complex permittivity in an treated area, i.e. knowledge of the complex permittivity of healthy and tumor tissue, is very important for example in the diagnosing of tumor regions in the human body (Choi et al., 2004) or in the design of thermo-therapeutic applicators which transform electromagnetic energy into thermal energy in the tissue (Williams et al., 2008). Permittivity is known as

$$
\varepsilon=\varepsilon_{0} \varepsilon_{c}
$$

where $\varepsilon_{0}$ is free space permittivity and $\varepsilon_{c}$ is complex relative permittivity. Complex relative permittivity can be written as

$$
\varepsilon_{c}=\varepsilon_{r}-j \varepsilon_{r} \tan \delta
$$

where $\varepsilon_{r}$ is real part of complex relative permittivity and $\tan \delta$ is loss factor.

\subsection{Methods of complex permittivity measurement}

The most commonly used method for measuring the complex permittivity is a method of measuring dielectric inserted into a dielectric waveguide. This method is very accurate, but unfortunately requires irreversible changes in the measured material. Another method used for measurement of complex permittivity is RLC measuring bridge. This method is very accurate but there are a few requirements on the quality of the interfaces of the measured material and the capacitor plates (Thompson, 1956). In most cases, this 
method is used as narrowband. The method of measurement in free space has its limitations in the demand for high-loss dielectrics measured. Electromagnetic wave through the material must be attenuated by at least $10 \mathrm{~dB}$. Otherwise, standing waves will be created, which contribute significantly to the inaccuracy of this method. The method of measurement in cavity resonators gives us results with good accuracy. On the other hand, it is difficult to produce precise machining of the resonator and the measured material inserted inside (Ramachandraiah et al., 1975). Latest often used method for measuring complex permittivity measurement method is the open end of the waveguide, in our case, the coaxial cable. This method can be considered as very accurate. Moreover, we can achieve a good repetition of the results when we maintain the phase stability of the measuring coaxial cable (Tanaba \& Joines, 1976). In this work, this application was selected for the main method for measuring complex permittivity of biological tissues because of its non-invasive nature.

\subsubsection{Principle of reflection method}

The reflection method represents measurement of reflection coefficient on the interface between two materials, on the open end of the coaxial line and the material under test. It is a well-known method for determining the dielectric parameters (Tanaba \& Joines, 1976). This method is based on the fact that the reflection coefficient of an open-ended coaxial line depends on the dielectric parameters of material under test which is attached to it. For calculating the complex permittivity from the measured reflection coefficient, it is useful to use an equivalent circuit of an open-ended coaxial line. The probe translates changes in the permittivity of a material under test into changes of the input reflection coefficient of the probe. The surface of the sample of material under test must be in perfect contact with the probe. The thickness of a measured sample must be at least twice of equivalent penetration depth of the electromagnetic wave. This assures that the waves reflected from the material under test interface are attenuated (Stuchly et al., 1994).

\subsection{Measurement probes}

For measurement probes, we have adapted the standard N and SMA RF connectors from which the parts for connecting to a panel were removed. The measurement probes can be described by the equivalent circuit consisting of the coupling capacitance between the inner and outer conductor out of the coaxial structure and radiating conductance which represents propagation losses (Popovic el al., 2005). These capacitance and conductance are frequency and permittivity dependent.

\subsubsection{N probe}

Probe for measuring the complex permittivity of biological tissues has been adapted from a standard RF connector for coaxial cable N-type connector, see Fig. 27. The original proposed range of application was $f_{\mathrm{BW}}=1 \mathrm{GHz}$, which together with the development of microwave technology is extended until today's $\mathrm{f}_{\mathrm{BW}}=12 \mathrm{GHz}$. $\mathrm{N}$ connector used in the construction of the probe is standard N $50 \Omega$ connector that can be used up to frequency $f=5 \mathrm{GHz}$. The measurements were kept with a high degree of the accuracy and repeatability. The measurement probe based on $\mathrm{N}$ connectors had significantly less bandwidth, and $\mathrm{f}_{1}=100 \mathrm{MHz}$ and $\mathrm{f}_{2}=1 \mathrm{GHz}$. 

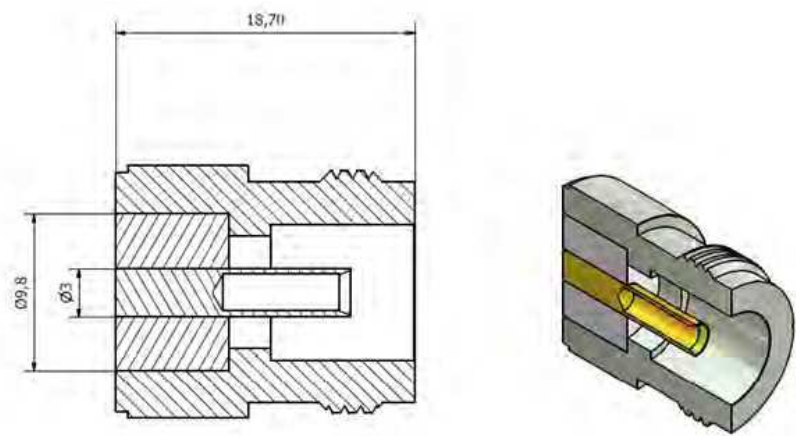

Fig. 27. Basic scheme of the $\mathrm{N}$ probe's dimensions

\subsubsection{SMA probe}

Probe for measuring the complex permittivity based on the SMA-connector was adapted from a standard $50 \Omega$ SMA connector which was developed around 1960 when it was required to create a miniaturized version of the $\mathrm{N}$ connector with a higher frequency range of application. Today SMA connectors operate in the band up to $\mathrm{f}=18 \mathrm{GHz}$. SMA connector used for the production of SMA probes, Fig. 28. shows low-cost, standard RF connectors with a usable bandwidth up to $\mathrm{f}=12 \mathrm{GHz}$. This solution is appropriate for the purposes of our measurements because the highest frequency measured is equal to $\mathrm{f}=2 \mathrm{GHz}$.
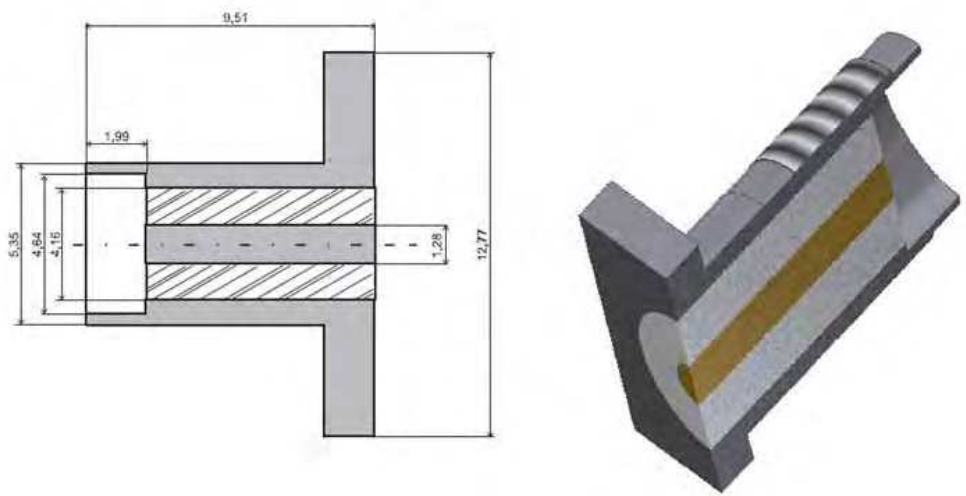

Fig. 28. Basic scheme of the SMA probe's dimensions

\subsection{Measurement setup}

A typical measurement setup using the reflection method on an open-ended coaxial line consists of the network analyzer, the coaxial probe and software. Our measurements were done with the aid of Agilent 6052 network analyzer in the frequency range from $300 \mathrm{MHz}$ to $3 \mathrm{GHz}$. First the calibration of the vector network analyzer is performed. Then the calibration using a reference material is done. Finally, the reflection coefficient of material under test is measured. The complex permittivity of material under test is evaluated by the MATLAB. 


\subsubsection{Measurement on inhomogeneous agar phantom}

The initial measurements on the inhomogeneous agar phantom were performed (Fig. 29.). As the simulations shows that the effective penetration depth in both cases, for $\mathrm{N}$ probe and SMA probe, is an interval of $4 \mathrm{~mm}$ to $2 \mathrm{~mm}$ depending on the frequency of measurement. Therefore, in this model of inhomogeneous agar phantom, strange dielectric immersion depths lies in the range from $4 \mathrm{~mm}$ to $1 \mathrm{~mm}$. Thus, the purpose of this measurement was to detect strange dielectrics that are covered with an agar layer in terms of simulating the electrical parameters of human skin and soft tissue. Inhomogeneous agar phantom was covered with a regular square grid (30 points $\times 30$ points), with the 900 points of measurement. Subsequently, measurements were taken at these points by the $\mathrm{N}$ probe and SMA-probe.

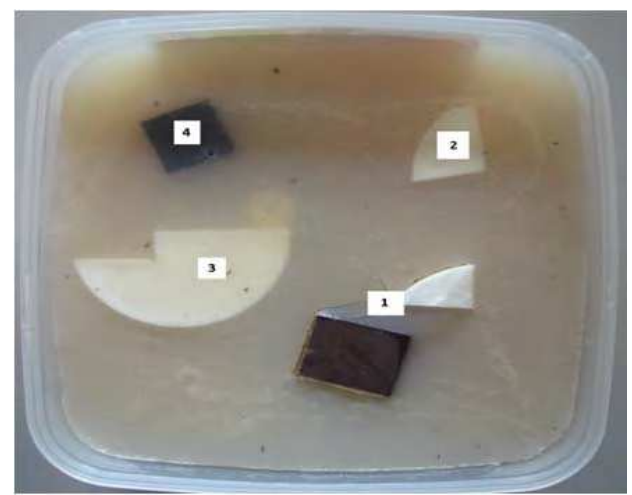

Fig. 29. Inhomogeneous agar phantom setup, the number shows the depth of strange dielectrics submersion

\subsubsection{Measurement on biological tissue}

The final verification of each experiment is necessary for practical usage. In our case, the experiment consisted of measuring the real biological tissue - the left upper limb of the first author (Fig. 30.). For this measurement, the SMA probe was used due to its ability to operate in higher bandwidth, and also because of its smaller size, and therefore a higher lateral resolution. The author's arm was marked with equidistant grid of measuring points, which then for-SMA probe measurements were carried out over a bandwidth of $\mathrm{f}_{\mathrm{MIN}}=300 \mathrm{MHz}$ to $\mathrm{f}_{\mathrm{MAX}}=2 \mathrm{GHz}$. Measurements were carried out very quickly with a delay of $1 \mathrm{~s}$ and $2 \mathrm{~s}$ at each measuring point. The data was saved (with the help of an assistant) in CSV data format in the flash drive connected to the vector analyzer. Frequency sweep was set at 1600 points at a bandwidth of $f_{\mathrm{MIN}}=300 \mathrm{MHz}$ to $\mathrm{f}_{\mathrm{MAX}}=2 \mathrm{GHz}$ and averaging was set to 16 in order to eliminate unwanted noise.

\subsection{Results}

The data presented here, obtained from measurements of the inhomogeneous phantom agar, are interpreted as a contour graph. Then there is the measurement on the left upper extremity of the author, which is displayed as contour graphs. All measurements were made in the band $\mathrm{f}_{\mathrm{MIN}}=300 \mathrm{MHz}$ to $\mathrm{f}_{\mathrm{MAX}}=2 \mathrm{GHz}$, from whom were selected three representative frequencies $\mathrm{f}_{1}=500 \mathrm{MHz}, \mathrm{f}_{2}=1 \mathrm{GHz}$ and $\mathrm{f}_{3}=2 \mathrm{GHz}$. 


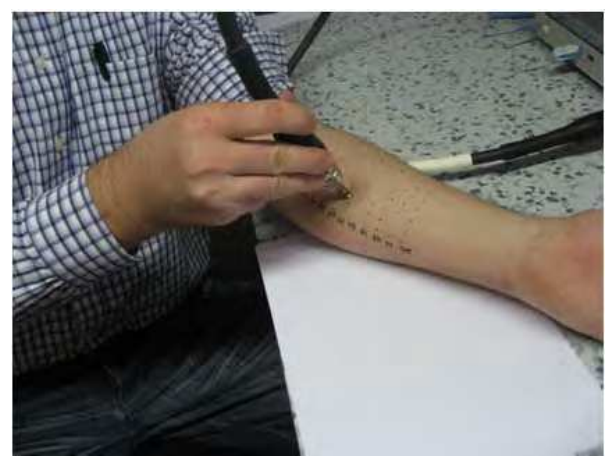

Fig. 30. Measurement on real biological tissue

\subsubsection{Results of measurement on inhomogeneous agar phantom}

For the measurement on inhomogeneous agar phantom was designed with a submerged object of Teflon $\varepsilon_{\mathrm{r}}=2.1$ and Bakelite $\varepsilon_{\mathrm{r}}=4.1$, immersed in a variable depth of $4 \mathrm{~mm}$ to $1 \mathrm{~mm}$ into the agar phantom. After solidify of the agar surface was applied spatially equidistant grid $(30 \times 30)$ with 900 data points.

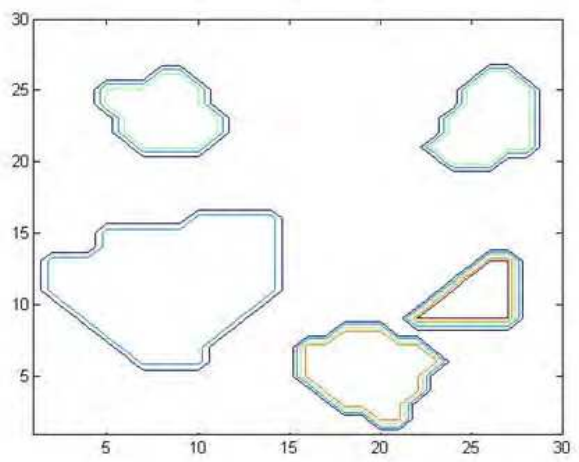

Fig. 31. Results of the measurement on inhomogeneous agar phantom (contour graph of real part of complex permittivity on frequency $\mathrm{f}=500 \mathrm{MHz}$ )

Measurements were performed by the SMA probe. Obtained values of the real part of complex permittivity have been rewritten into MATLAB and displayed as a contour graph image, see Fig. 31. The results of the measurement on the inhomogeneous agar phantom have shown the potential of imaging usability of this method for further investigations on real living tissue.

\subsubsection{Results of measurement on biological tissue}

Testing the imaging potential for complex permittivity measurement of biological tissue was finally performed on the left upper extremity of the author, see Fig. 30. SMA probe was used for this measurement. For a small separation of complex permittivity of biological tissue, these data are displayed only in the contour graph. It is very difficult to assess tissue formations without further visual assessment, using MRI and subsequent processing of data segmentation algorithms, but the Fig. 32a,b. shows that contours of real part of complex permittivity during 
the various measurements still occur in the same places. Among the limitations of the method, we can mention a small depth of penetration shown in Fig. 32c) at frequency $\mathrm{f}=2000 \mathrm{MHz}$; as a result, we can register only the layer of skin. The actual depth of penetration can be improved by using of probes based on the waveguide thus having a greater radiating aperture that would better achieve the quasi-plane waves, but the loss of lateral resolution of the probe. Overcoming this eternal compromise can be achieved through a combination of two probes for a single measurement, one with an emphasis on greater penetration depth and the second for acceptable lateral resolution. When comparing the results of this measurement shown in Fig. 32., we can find among them a high degree of correlation pointing out that in real biological tissue this measuring method will able to detect coherent tissue structure having the same value of the real part of complex permittivity.

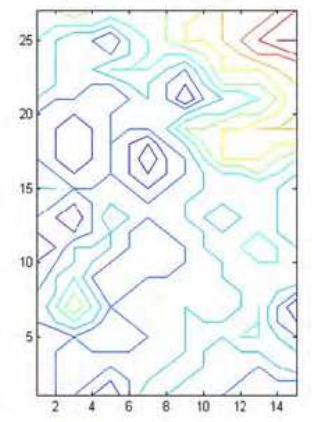

a)

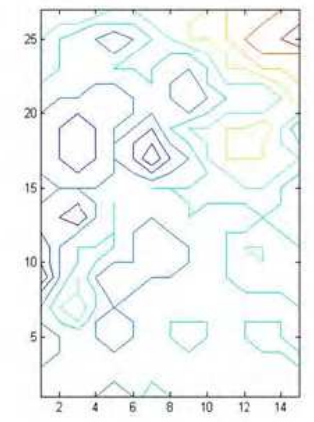

b)

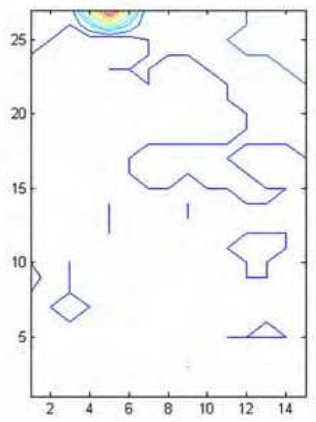

c)

Fig. 32. Results of the measurement on real biological tissue (contour graph of real part of complex permittivity on frequency a) $\mathrm{f} 1=500 \mathrm{MHz}, \mathrm{b}$ ) $\mathrm{f} 2=1 \mathrm{GHz}, \mathrm{c}$ ) $\mathrm{f} 3=2 \mathrm{GHz}$

\subsection{Evaluation}

Measurements on inhomogeneous agar phantom showed the potential of this imaging method, because the strange dielectrical materials were detectable beneath the surface of agar. Based on this result, the measurement of the left upper extremity of the author was performed, and the contour map of the real part of complex permittivity shown the detectable difference between the tissues. The contour graphs of the real part of complex permittivity of biological tissue give us the hope for the future development that this method may be used as a imaging method. After proving more interaction on the pathological changes in tissues, such as growth of tumor tissue, we can consider this method for diagnostic purposes.

\section{Conclusions}

Research described

\section{Acknowledgment}

Research described in this chapter was supported by Grant Agency of the Czech Republic, projects: 102/08/H081:,,Non-standard application of physical fields - analogy, modelling, verification and simulation" and P102/11/0649: "Research and measurements of signals 
generated by nanoparticles". Further by research program MSM6840770012: "Transdisciplinary Research in the Area of Biomedical Engineering II" of the CTU in Prague, sponsored by the Ministry of Education, Youth and Sports of the Czech Republic.

\section{References}

Able Software Corp. (2011). 3D-DOCTOR, 3D Imaging, Modeling, Rendering and Measurement Software, Available from: http://www.ablesw.com/3d-doctor

Bolmsjö, M.; Wagrell, L.; Hallin, A.; Eliasson, T.; Erlandsson, B.E. \& Mattiasson, A. (1996). The heat is on - but how? A comparison of TUMT devices. The Journal of Urology, Vol.78, pp. 564-572

Choi, J.; Cho, Y. Lee, J. Yim, B.; Kang, K. \& et al. (2004). Microwave detection of metastasized breast cancer cells in the lymph node, potential application for sentinel lymphadenectomy, In: Breast Cancer Research and Treatment, Vol.86, No.2, pp. 107-115, Springer, ISSN 0167-6806

De la Rosette, J.; D'Ancona, F. \& Debruyne, F. (February 1997). Current status of thermotherapy of the prostate, The Journal of Urology, Vol.157, pp. 430-438

Franconi, C.; Vrba, J. \& Montecchia, F. (1993). $27 \mathrm{MHz}$ Hybrid Evanescent-Mode Applicators. International Journal of Hyperthermia, Vol.9, No.5, pp. 655 - 673

Hand, J. \& James, J. R. (1986). Physical Techniques in Clinical Hyperthermia. Wiley, New York

Hippel, A. (1954). Dielectric Materials and Applications. Technology Press of MIT, Cambridge, 1954, pp. 71-75

Popovic, D.; McCartney, L.; Beasley, C.; Lazebnik, M.; Okoniewski, M.; Hagness, S.C. \& Booske, J.H. (2005). Precision open-ended coaxial probes for in vivo and ex vivo dielectric spectroscopy of biological tissues at microwave frequencies. IEEE Transactions on Microwave Theory and Techniques, Vol.53, No.5, pp. 1713-1722, ISSN 0018-9480

Ramachandraiah, M. S. \& Decreton, M. C. (December 1975). A Resonant Cavity Approach for the Nondestructive Determination of Complex Permittivity at Microwave Frequencies. Instrumentation and Measurement, IEEE Transactions on Instrumentation and Measurement, Vol.24, No.4, pp.287-291, ISSN 0018-9456

Tanaba, E. \& Joines, W. T. (1976). A nondestructive method for measurement of the complex permittivity of dielectric materials at microwave frequency using an open-ended coaxial line resonator. IEEE Trans. on Measurement and Instrumentation, Vol.25, pp. 222-226, ISSN 0018-9480

Thompson, A. M. (November 1956). A bridge for the measurement of permittivity. Proceedings of the IEE - Part B: Radio and Electronic Engineering, Vol.103, No.12, pp. 704-707

Vrba, J.; Franconi, C. \& Lapeš, M. (1996). Theoretical Limits for the Penetration Depth of the Intracavitary Applicat. International Journal of Hyperthermia, No.6., pp.737 - 742

Vrba, J.; Franconi, C.; Montecchia, F. \& Vannucci, I. (May 1993). Evanescent-Mode Applicators for Hyperthermia. IEEE Trans.on Biomedical Engineering, Vol.40, No.5, pp. 397-407

Vrba, J.; Lapeš, M. \& Oppl, L. (1999). Technical aspects of microwave thermotherapy. Bioelectrochemistry and Bioenergetics, Vol.48, pp. 305 - 309

Williams, T. C.; Sill, J.M. \& Fear, E.C. (May 2008). Breast surface estimation for radar-based breast imaging systems. IEEE Transactions on Biomedical Engineering, Vol.55, No.6, pp. 1678-1686, ISSN 0018-9294 


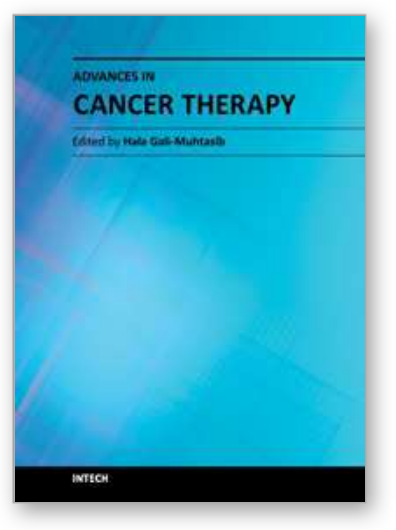

\author{
Advances in Cancer Therapy \\ Edited by Prof. Hala Gali-Muhtasib
}

ISBN 978-953-307-703-1

Hard cover, 568 pages

Publisher InTech

Published online 21, November, 2011

Published in print edition November, 2011

The book "Advances in Cancer Therapy" is a new addition to the Intech collection of books and aims at providing scientists and clinicians with a comprehensive overview of the state of current knowledge and latest research findings in the area of cancer therapy. For this purpose research articles, clinical investigations and review papers that are thought to improve the readers' understanding of cancer therapy developments and/or to keep them up to date with the most recent advances in this field have been included in this book. With cancer being one of the most serious diseases of our times, I am confident that this book will meet the patients', physicians' and researchers' needs.

\title{
How to reference
}

In order to correctly reference this scholarly work, feel free to copy and paste the following:

Jaroslav Vorliček, Barbora Vrbova and Jan Vrba (2011). Prospective Applications of Microwaves in Medicine, Advances in Cancer Therapy, Prof. Hala Gali-Muhtasib (Ed.), ISBN: 978-953-307-703-1, InTech, Available from: http://www.intechopen.com/books/advances-in-cancer-therapy/prospective-applications-of-microwavesin-medicine

\section{INTECH}

open science | open minds

\section{InTech Europe}

University Campus STeP Ri Slavka Krautzeka 83/A 51000 Rijeka, Croatia

Phone: +385 (51) 770447

Fax: +385 (51) 686166 www.intechopen.com

\section{InTech China}

Unit 405, Office Block, Hotel Equatorial Shanghai No.65, Yan An Road (West), Shanghai, 200040, China 中国上海市延安西路65号上海国际贵都大饭店办公楼 405 单元 Phone: +86-21-62489820

Fax: $+86-21-62489821$ 
(C) 2011 The Author(s). Licensee IntechOpen. This is an open access article distributed under the terms of the Creative Commons Attribution 3.0 License, which permits unrestricted use, distribution, and reproduction in any medium, provided the original work is properly cited. 